Article

\title{
On Assignment of the Upper Bohl Exponent for Linear Time-Invariant Control Systems in a Hilbert Space by State Feedback
}

\author{
Vasilii Zaitsev ${ }^{*}+\boldsymbol{t}^{+}$and Marina Zhuravleva ${ }^{\dagger}$ \\ Laboratory of Mathematical Control Theory, Udmurt State University, Izhevsk 426034, Russia; \\ mrnzo@yandex.ru \\ * Correspondence: verba@udm.ru \\ t These authors contributed equally to this work.
}

Received: 18 May 2020; Accepted: 14 June 2020; Published: 17 June 2020

check for updates

\begin{abstract}
We consider a linear continuous-time control system with time-invariant linear bounded operator coefficients in a Hilbert space. The controller in the system has the form of linear state feedback with a time-varying linear bounded gain operator function. We study the problem of arbitrary assignment for the upper Bohl exponent by state feedback control. We prove that if the open-loop system is exactly controllable then one can shift the upper Bohl exponent of the closed-loop system by any pregiven number with respect to the upper Bohl exponent of the free system. This implies arbitrary assignability of the upper Bohl exponent by linear state feedback. Finally, an illustrative example is presented.
\end{abstract}

Keywords: linear control system; Hilbert space; state feedback control; exact controllability; upper Bohl exponent

MSC: 34D08; 34A35; 93C05

\section{Introduction}

Consider a linear control system:

$$
\dot{x}(t)=A(t) x(t)+B(t) u(t), \quad t \in \mathbb{R} .
$$

Here $x \in \mathfrak{X}$ and $u \in \mathfrak{U}$ are the state and control vectors respectively, $\mathfrak{X}$ and $\mathfrak{U}$ are some finite-dimensional or infinite-dimensional Banach spaces. Suppose that the controller in system (1) has the form of linear static state feedback $u(t)=U(t) x(t)$. The closed-loop system has the form:

$$
\dot{x}(t)=(A(t)+B(t) U(t)) x(t), \quad t \in \mathbb{R} .
$$

Now we consider the elements of the gain operator $U(t)$ as controlling parameters. The problems of control over the asymptotic behavior of solutions to systems (2) by means of elements of gain operator $U(t)$ (in particular, the problem of stabilization for system (2)) belong to the classical problems of control theory. First results relate to stationary systems in finite-dimensional spaces. It was proved for complex [1] and real [2] finite-dimensional $\left(\mathfrak{X}=\mathbb{R}^{n}, \mathfrak{U}=\mathbb{R}^{m}\right)$ time-invariant $(A(t) \equiv A, B(t) \equiv B)$ systems that the condition of complete controllability of system (1) is necessary and sufficient for the arbitrary assignment of the eigenvalue spectrum $\lambda_{1}, \ldots, \lambda_{n}$ of the closed-loop system (2) by means of time-invariant $(U(t) \equiv U)$ feedback. This implies, in particular, stabilizability of (2) by means of $U(t) \equiv U$. First results for time-varying periodic systems in finite-dimensional spaces were obtained 
in [3]: It was proved that the complete controllability of system (1) is necessary and sufficient for the arbitrary assignment of the characteristic multipliers $\rho_{1}, \ldots, \rho_{n}$ of the closed-loop system (2) by means of periodic feedback. For time-varying non-periodic systems in finite-dimensional spaces, first results on stabilization were obtained in [4-6]. A transformation reducing system (2) to a canonical (block)-Frobenius form was used, which allows one to solve the eigenvalue assignment problem. However, rather restrictive conditions on the smoothness and boundedness of the coefficients of system (1) are required there. These conditions were weakened in [7] to the condition of uniform complete controllability in the sense of Kalman [8], and, on the basis of this property, sufficient conditions for exponential stabilization of system (2) were obtained. The proof of exponential stability is carried out using the second Lyapunov method (the Lyapunov function method).

In the framework of the first Lyapunov method of studying systems of differential equations in finite-dimensional spaces, a natural generalization of the concept of eigenvalue spectrum for non-stationary systems is the spectrum of Lyapunov exponents (see [9-11]). In addition to Lyapunov exponents, other Lyapunov invariants are known (that is, characteristics that do not change under the Lyapunov transformation, see [12]), which characterize the asymptotic behavior of solutions to a linear system of differential equations, for example, the Bohl exponents, the central (Vinograd) exponents, the exponential (Izobov) exponents, etc. In a series of studies [13-17], the results on arbitrary assignability of Lyapunov exponents and other Lyapunov invariants for system (2) in finite-dimensional spaces were proved, based on the property of uniform complete controllability in the sense of Kalman. In recent studies [18-23], these results have been partially extended to discrete-time systems. In finite-dimensional spaces, the Lyapunov exponents, the Bohl exponents, and other Lyapunov invariants were studied, for example in [24-26] for continuous-time systems and in [27-33] for discrete-time systems.

A large number of papers are devoted to stabilization problems of system (2) in infinite-dimensional spaces. We note here the studies [34-41]. Properties of the spectrum for systems in infinite-dimensional spaces were studied in [42-44].

In this paper, we studied the problem of arbitrary assignment of the upper Bohl exponent for continuous-time systems in an infinite-dimensional Hilbert space. The brief outline of the paper is as follows. In Section 2, some notations, definitions, and preliminary results are given and the concepts used throughout the paper are defined, as well as some basic theories, methods, and techniques. In Section 3, we analyze the problem of arbitrary assignment of the upper Bohl exponent by means of linear state feedback with a time-varying linear bounded gain operator function for linear time-invariant control system in a Hilbert space with bounded operator coefficients and prove that the property of exact controllability of the open-loop system is sufficient for arbitrary assignability of the upper Bohl exponent of the closed-loop system. Section 4 provides an illustrative example that emphasizes the theory. In Section 5, we revise the results obtained in the paper and also showcase future developments of the theory.

\section{Notations, Definitions, and Preliminary Results}

Let $\mathfrak{X}$ be a Banach space, $\mathfrak{X}^{*}$ be dual to $\mathfrak{X}$. By $\mathcal{L}\left(\mathfrak{X}_{1}, \mathfrak{X}_{2}\right)$ we denote a Banach space of linear bounded operators $A: \mathfrak{X}_{1} \rightarrow \mathfrak{X}_{2}$. If $A \in \mathcal{L}\left(\mathfrak{X}_{1}, \mathfrak{X}_{2}\right)$, then $A^{*} \in \mathcal{L}\left(\mathfrak{X}_{2}^{*}, \mathfrak{X}_{1}^{*}\right)$ is its adjoint operator. By $I: \mathfrak{X} \rightarrow \mathfrak{X}$ denote the identity operator.

Consider a linear system of differential equations:

$$
\dot{x}(t)=A(t) x(t), \quad t \in \mathbb{R}, \quad x \in \mathfrak{X} .
$$

We suppose that the following conditions hold:

(a) $A(t) \in \mathcal{L}(\mathfrak{X}, \mathfrak{X})$ for any $t \in \mathbb{R}$;

(b) The function $\mathbb{R} \ni t \mapsto A(t) \in \mathcal{L}(\mathfrak{X}, \mathfrak{X})$ is piecewise continuous;

(c) $\sup _{t \in \mathbb{R}}\|A(t)\|=a<+\infty$. 
By a solution of system (3) we will understand, by definition, a solution of the integral equation:

$$
x(t)=x_{0}+\int_{t_{0}}^{t} A(s) x(s) d s,
$$

where

$$
x\left(t_{0}\right)=x_{0} .
$$

Due to conditions imposed on $A(\cdot)$, a solution (4) of (3) is a continuous, piecewise continuously differentiable function and satisfies (3) almost everywhere ([45], Ch. III, Sect. 1.1, 1.2).

By $\Phi(t, \tau)$ denote the evolution operator of system (3) ([45], Ch. III, Sect. 1, p. 100) that is the solution of the operator system:

$$
\frac{d X}{d t}=A(t) X, \quad X(\tau)=I .
$$

By using the operator $\Phi(t, \tau)$, the solution ot the initial value problem (3), (5) can be expressed by the formula $x(t)=\Phi\left(t, t_{0}\right) x_{0}$.

The evolution operator $\Phi(t, \tau)$ has the following properties ([45], Ch. III, Sect. 1, p. 101):
(A) $\Phi(t, t)=I$;
(B) $\Phi(t, s) \Phi(s, \tau)=\Phi(t, \tau)$;
(C) $\Phi(t, \tau)=[\Phi(\tau, t)]^{-1}$;

$$
\text { (D) } \quad \exp \left(-\int_{s}^{t}\|A(\tau)\| d \tau\right) \leq\left\|\Phi^{ \pm 1}(t, s)\right\| \leq \exp \left(\int_{s}^{t}\|A(\tau)\| d \tau\right), \quad s \leq t
$$

(see [45], Ch. III, Sect. 2, (2.25)). It follows from property $(D)$ and condition $(c)$ that:

$$
e^{-a(t-s)} \leq\left\|\Phi^{ \pm 1}(t, s)\right\| \leq e^{a(t-s)}, \quad s \leq t .
$$

Definition 1. The upper Bohl exponent ([45], Ch. III, Sect.4) of system (3) is the number:

$$
\varkappa(A)=\varlimsup_{\tau, s \rightarrow+\infty} \frac{\ln \|\Phi(\tau+s, \tau)\|}{s} .
$$

The upper Bohl exponent of system (3) characterizes asymptotic behavior of solutions of (3): The condition $\varkappa(A)<0$ is necessary and sufficient for uniform exponential stability of all solutions to system (3). Due to the condition (c), the upper Bohl exponent of system (3) is finite ([45], Ch. III, Sect. 4, Theorem 4.3).

Let us apply the $\lambda$-transformation ([9], p. 249), ([45], Ch. III, Sect. 4, p. 124) to system (3) that is adding the disturbance $\lambda I$ to the operator $A(t)$ and consider the disturbed system:

$$
\dot{z}(t)=(A(t)+\lambda I) z(t), \quad t \in \mathbb{R}, \quad z \in \mathfrak{X} .
$$

By $\Psi(t, \tau)$ denote the evolution operator of system (7).

Lemma 1. For any $t, \tau \in \mathbb{R}$ the following equality holds:

$$
\Psi(t, \tau)=e^{\lambda(t-\tau)} \Phi(t, \tau) .
$$

Proof. Calculating the derivative of the right-hand side of (8), we obtain:

$$
\frac{d}{d t}\left(e^{\lambda(t-\tau)} \Phi(t, \tau)\right)=\lambda e^{\lambda(t-\tau)} \Phi(t, \tau)+e^{\lambda(t-\tau)} A(t) \Phi(t, \tau)=(A(t)+\lambda I) e^{\lambda(t-\tau)} \Phi(t, \tau) .
$$

Next,

$$
\left.e^{\lambda(t-\tau)} \Phi(t, \tau)\right|_{t=\tau}=I
$$


It follows from (9) and (10) that $e^{\lambda(t-\tau)} \Phi(t, \tau)$ is the evolution operator of (7). Due to the uniqueness of the evolution operator, $e^{\lambda(t-\tau)} \Phi(t, \tau)$ coincides with $\Psi(t, \tau)$.

Lemma 2. $\varkappa(A+\lambda I)=\varkappa(A)+\lambda$.

Proof. By using Lemma 1, we obtain:

$$
\begin{gathered}
\varkappa(A+\lambda I)=\varlimsup_{\tau, s \rightarrow+\infty} \frac{\ln \|\Psi(\tau+s, \tau)\|}{s}=\varlimsup_{\tau, s \rightarrow+\infty} \frac{\ln \left\|e^{\lambda s} \Phi(\tau+s, \tau)\right\|}{s} \\
=\varlimsup_{\tau, s \rightarrow+\infty} \frac{\ln \left(e^{\lambda s}\|\Phi(\tau+s, \tau)\|\right)}{s}=\varlimsup_{\tau, s \rightarrow+\infty} \frac{\ln e^{\lambda s}+\ln \|\Phi(\tau+s, \tau)\|}{s} \\
=\varlimsup_{\tau, s \rightarrow+\infty}\left(\frac{\lambda s}{s}+\frac{\ln \|\Phi(\tau+s, \tau)\|}{s}\right)=\varlimsup_{\tau, s \rightarrow+\infty}\left(\lambda+\frac{\ln \|\Phi(\tau+s, \tau)\|}{s}\right) \\
=\lambda+\varlimsup_{\tau, s \rightarrow+\infty} \frac{\ln \|\Phi(\tau+s, \tau)\|}{s}=\lambda+\varkappa(A) .
\end{gathered}
$$

Let us consider another linear system of differential equations:

$$
\dot{y}(t)=C(t) y(t), \quad t \in \mathbb{R}, \quad y \in \mathfrak{X} .
$$

Suppose that the operator function $C(t)$ also satisfies conditions $(a),(b),(c)$, i.e., $C(t) \in \mathcal{L}(\mathfrak{X}, \mathfrak{X})$ $\forall t \in \mathbb{R}, C(\cdot)$ is piecewise continuous, and $\sup _{t \in \mathbb{R}}\|C(t)\|=c<+\infty$. By $\Theta(t, \tau)$ denote the evolution operator of system (11). Because of conditions imposed on $C(\cdot)$, we have the inequality:

$$
e^{-c(t-s)} \leq\left\|\Theta^{ \pm 1}(t, s)\right\| \leq e^{c(t-s)}, \quad s \leq t .
$$

Definition 2. Systems (3) and (11) are called kinematically similar on $\mathbb{R}$ ([45], Ch.IV, Sect. 2) if it is possible to establish between the totalities of all solutions of these systems a one-to-one correspondence:

$$
y(t)=L(t) x(t), \quad t \in \mathbb{R},
$$

where $L(t)$ is a bounded linear operator function with a bounded inverse:

$$
\|L(t)\| \leq d_{1}, \quad\left\|L^{-1}(t)\right\| \leq d_{2}, \quad t \in \mathbb{R} .
$$

The following criterion holds (see [45], Ch. IV, Sect. 2, Lemma 2.1, (a)).

Lemma 3. Systems (3) and (11) are kinematically similar on $\mathbb{R}$ if and only if there exists an operator function $\mathbb{R} \ni t \mapsto L(t) \in \mathcal{L}(\mathfrak{X}, \mathfrak{X})$ satisfying (13) and such that the evolution operators of the systems are connected by the relation:

$$
\Theta(t, \tau) L(\tau)=L(t) \Phi(t, \tau)
$$

Lemma 4 (see [45], Ch. IV, Sect. 2, Theorem 2.1). If systems (3) and (11) are kinematically similar on $\mathbb{R}$, then $\varkappa(A)=\varkappa(C)$.

Let us state sufficient conditions for kinematical similarity of systems (3) and (11) on $\mathbb{R}$ analogous to the corresponding conditions in a finite-dimensional space (see, e.g., [46]).

Lemma 5. Suppose that the operator functions $A(t)$ and $C(t)$ satisfy conditions $(a),(b)$, and $(c)$, and there exists a sequence $\left\{t_{i}\right\}_{i \in \mathbb{Z}} \subset \mathbb{R}$ such that $0<\rho_{1} \leq t_{i+1}-t_{i} \leq \rho_{2}$ and $\Phi\left(t_{i+1}, t_{i}\right)=\Theta\left(t_{i+1}, t_{i}\right)$ for all $i \in \mathbb{Z}$. Then systems (3) and (11) are kinematically similar on $\mathbb{R}$. 
Proof. By using the group property $(B)$ of evolution operators, we obtain for all $j>i$ :

$$
\Phi\left(t_{j}, t_{i}\right)=\Phi\left(t_{j}, t_{j-1}\right) \cdots \Phi\left(t_{i+1}, t_{i}\right)=\Theta\left(t_{j}, t_{j-1}\right) \cdots \Theta\left(t_{i+1}, t_{i}\right)=\Theta\left(t_{j}, t_{i}\right) .
$$

By $(C),(15)$ holds for any $i, j \in \mathbb{Z}$. Let us construct the operator function:

$$
L(t)=\Theta\left(t, t_{0}\right) \Phi\left(t_{0}, t\right) .
$$

By (15), we have $L\left(t_{i}\right)=I, i \in \mathbb{Z}$. Next, by (16), we have:

$$
\begin{aligned}
\Theta(t, \tau) L(\tau) & =\Theta(t, \tau) \Theta\left(\tau, t_{0}\right) \Phi\left(t_{0}, \tau\right)=\Theta\left(t, t_{0}\right) \Phi\left(t_{0}, \tau\right), \\
L(t) \Phi(t, \tau) & =\Theta\left(t, t_{0}\right) \Phi\left(t_{0}, t\right) \Phi(t, \tau)=\Theta\left(t, t_{0}\right) \Phi\left(t_{0}, \tau\right) .
\end{aligned}
$$

Hence, (14) is fulfilled. Let us prove that (13) is satisfied.

Let $t \in \mathbb{R}$ be an arbitrary number. Then, since $t_{i+1}-t_{i} \geq \rho_{1}$, there exists an $i_{0} \in \mathbb{Z}$ such that $t \in\left[t_{i_{0}}, t_{i_{0}+1}\right]$. In this case, $t-t_{i_{0}} \leq \rho_{2}$. We have:

$$
\begin{gathered}
L(t)=\Theta\left(t, t_{0}\right) \Phi\left(t_{0}, t\right)=\Theta\left(t, t_{i_{0}}\right) \Theta\left(t_{i_{0}}, t_{0}\right) \Phi\left(t_{0}, t_{i_{0}}\right) \Phi\left(t_{i_{0}}, t\right) \\
=\Theta\left(t, t_{i_{0}}\right) L\left(t_{i_{0}}\right) \Phi\left(t_{i_{0}}, t\right)=\Theta\left(t, t_{i_{0}}\right) \Phi\left(t_{i_{0}}, t\right) .
\end{gathered}
$$

So, $L^{-1}(t)=\Phi\left(t, t_{i_{0}}\right) \Theta\left(t_{i_{0}}, t\right)$. Then, taking (6) and (12) into account, we obtain:

$$
\begin{gathered}
\|L(t)\| \leq\left\|\Theta\left(t, t_{i_{0}}\right)\right\| \cdot\left\|\Phi\left(t_{i_{0}}, t\right)\right\| \leq e^{c\left(t-t_{i_{0}}\right)} e^{a\left(t-t_{i_{0}}\right)} \leq e^{(a+c) \rho_{2}}=: d_{1}, \\
\left\|L^{-1}(t)\right\| \leq\left\|\Phi\left(t, t_{i_{0}}\right)\right\| \cdot\left\|\Theta\left(t_{i_{0}}, t\right)\right\| \leq e^{a\left(t-t_{i_{0}}\right)} e^{c\left(t-t_{i_{0}}\right)} \leq e^{(a+c) \rho_{2}}=: d_{2} .
\end{gathered}
$$

Hence, (13) holds. By Lemma 3, the lemma is proved.

Consider a linear control system:

$$
\dot{x}(t)=A(t) x(t)+B(t) u(t), \quad t \in \mathbb{R} .
$$

Here $x \in \mathfrak{X}, u \in \mathfrak{U} ; \mathfrak{X}, \mathfrak{U}$ are Banach spaces; $A(t)$ satisfies conditions $(a),(b),(c) ; \forall t \in \mathbb{R}$ $B(t) \in \mathcal{L}(\mathfrak{U}, \mathfrak{X})$, the function $t \mapsto B(t)$ is piecewise continuous, and sup $\|B(t)\|<+\infty$. Admissible controllers for (17) on some finite interval $\left[t_{0}, t_{1}\right]$ are functions $u(\cdot) \in L_{p}\left(\left[t_{0}, t_{1}\right], \mathfrak{U}\right), p \geq 1$. For each admissible controller $u(\cdot)$, there is a unique solution of the initial value problem (17), (5) (see ([45], Ch. III, Sect. 1, (1.19)), [47]), determined by the formula:

$$
x(t)=\Phi\left(t, t_{0}\right) x_{0}+\int_{t_{0}}^{t} \Phi(t, \tau) B(\tau) u(\tau) d \tau .
$$

Here $\Phi(t, \tau)$ is the evolution operator of the corresponding free system (3). We consider a control system (17) without imposing any geometric constraints on the control or on the state.

Definition 3 (see [47]). System (17) is called exactly controllable on $[0, \vartheta]$ if for any $x_{0}, x_{1} \in \mathfrak{X}$ there exists an admissible controller $u(t), t \in[0, \vartheta]$, steering the solution of (17) from $x(0)=x_{0}$ to $x(\vartheta)=x_{1}$.

Suppose that the controller in system (17) has the form of the linear state feedback:

$$
u(t)=U(t) x(t)
$$


where $U(t) \in \mathcal{L}(\mathfrak{X}, \mathfrak{U}) \forall t \in \mathbb{R}, U(\cdot)$ is piecewise continuous, and $\sup _{t \in \mathbb{R}}\|U(t)\|<+\infty$. We say that the gain operator function $U(\cdot)$ satisfying these conditions is admissible. The closed-loop system has the form:

$$
\dot{x}(t)=(A(t)+B(t) U(t)) x(t) .
$$

By $\Phi_{U}(t, \tau)$ we denote the evolution operator of system (19).

Definition 4. We say that system (17) admits a $\lambda$-transformation if there exists a constant $\sigma>0$ such that, for any $\lambda \in \mathbb{R}$, there exists an admissible gain operator function $U(\cdot)$ ensuring that the evolution operator $\Phi_{U}(t, \tau)$ of system (19) satisfies the relation:

$$
\Phi_{U}((k+1) \sigma, k \sigma)=e^{\lambda \sigma} \Phi((k+1) \sigma, k \sigma)
$$

for all $k \in \mathbb{Z}$.

This definition was given in [13] for systems (17) in finite-dimensional spaces (see also [48]). It is related to the definition of a $\lambda$-transformation of system (3).

Remark 1. It follows from (20) that, for the evolution operator $\Phi_{U}(t, s)$ of system (19), the relation $\Phi_{U}(k \sigma, \ell \sigma)=e^{\lambda(k-\ell) \sigma} \Phi(k \sigma, \ell \sigma)$ holds that is similar to (8) but is fulfilled on the set $\{k \sigma, k \in \mathbb{Z}\} \subset \mathbb{R}$.

Theorem 1. Suppose that system (17) admits a $\lambda$-transformation. Then, for any $\lambda \in \mathbb{R}$, there exists an admissible gain operator function $U(\cdot)$ such that the closed-loop system (19) and system (7) are kinematically similar on $\mathbb{R}$.

Proof. It follows from (20) and (8) that, for all $k \in \mathbb{Z}$, the following equalities hold:

$$
\Phi_{U}\left(t_{k+1}, t_{k}\right)=\Psi\left(t_{k+1}, t_{k}\right)
$$

where $\Psi(t, s)$ is the evolution operator of system (7) and $t_{k}=k \sigma$. Now, applying Lemma 5 to systems (19) and (7), where $\rho_{1}=\rho_{2}=\sigma$, we obtain what is required.

Definition 5. We say that the upper Bohl exponent of system (17) is arbitrarily assignable by linear state feedback (18) if for any $\mu \in \mathbb{R}$ there exists an admissible gain operator function $U(\cdot)$ such that, for the closed-loop system (19),

$$
\varkappa(A+B U)=\mu .
$$

The corresponding definition in finite-dimensional spaces was given in [13] (see also [48]) for the upper (and lower) central (and Bohl) exponents.

\section{Main Results}

Consider a time-invariant control system (17):

$$
\dot{x}(t)=A x(t)+B u(t), \quad t \in \mathbb{R} .
$$

Here $x \in \mathfrak{X}, u \in \mathfrak{U} ; \mathfrak{X}$ and $\mathfrak{U}$ are separable Hilbert spaces; $A \in \mathcal{L}(\mathfrak{X}, \mathfrak{X}), B \in \mathcal{L}(\mathfrak{U}, \mathfrak{X}) ; a:=\|A\|$, $b:=\|B\|$. For Hilbert spaces $\mathfrak{H}_{1}, \mathfrak{H}_{2}$, we suppose that, if $F \in \mathcal{L}\left(\mathfrak{H}_{1}, \mathfrak{H}_{2}\right)$, then $F^{*} \in \mathcal{L}\left(\mathfrak{H}_{2}, \mathfrak{H}_{1}\right)$, i.e., we identify $\mathfrak{H}_{i}^{*}$ with $\mathfrak{H}_{i}$. By $\langle\cdot, \cdot\rangle$ denote the scalar product (in the corresponding space). If $F^{*}=F \in \mathcal{L}(\mathfrak{X}, \mathfrak{X})$, then the inequality $F \geq \alpha I$ means, by definition, that $\langle F x, x\rangle \geq \alpha\|x\|^{2}$ for all $x \in \mathfrak{X}$. 
The evolution operator of the corresponding free system:

$$
\dot{x}(t)=A x(t), \quad t \in \mathbb{R},
$$

has the form $\Phi(t, \tau)=\exp (A(t-\tau))$. Let us denote $\Phi(t):=\Phi(t, 0)=\exp (A t)$.

Let us construct the controllability gramian $Q(\vartheta): \mathfrak{X} \rightarrow \mathfrak{X}, \vartheta>0$ (see ([49], Definition 4.1.3), ([50], Part IV, Ch. 2, Sect. 2.2, (2.9))):

$$
Q(\vartheta) x=\int_{0}^{\vartheta} \Phi(s) B B^{*} \Phi^{*}(s) x d s .
$$

We have $Q(\vartheta) \in \mathcal{L}(\mathfrak{X}, \mathfrak{X})$ (see [49], Lemma 4.1.4), $Q(\vartheta)=Q^{*}(\vartheta)$, and

$$
\langle Q(\vartheta) x, x\rangle=\int_{0}^{\vartheta}\left\|B^{*} \Phi^{*}(s) x\right\|^{2} d s \geq 0, \quad x \in \mathfrak{X}
$$

(see [50], Part IV, Ch. 2, Sect. 2.2, (2.10)). By replacing $s$ by $\vartheta-t$ in (22), we obtain that:

$$
Q(\vartheta)=\int_{0}^{\vartheta} \Phi(\vartheta-t) B B^{*} \Phi^{*}(\vartheta-t) d t
$$

Lemma 6. $\|Q(\vartheta)\| \leq \vartheta e^{2 a \vartheta} b^{2}$.

Proof. It follows from $(D)$ that:

$$
e^{-a \vartheta} \leq\left\|\Phi^{ \pm 1}(t)\right\| \leq e^{a \vartheta}, \quad t \in[0, \vartheta] .
$$

Moreover, since $\Phi(t) \in \mathcal{L}(\mathfrak{X}, \mathfrak{X})$, we have $\left\|\Phi^{*}(t)\right\|=\|\Phi(t)\|$ (see [49], Lemma A.3.41). Thus,

$$
e^{-a \vartheta} \leq\left\|\left(\Phi^{*}(t)\right)^{ \pm 1}\right\| \leq e^{a \vartheta}, \quad t \in[0, \vartheta] .
$$

Similarly, $\left\|B^{*}\right\|=\|B\|=b$. By using (22), (24), and (25), we obtain:

$$
\|Q(\vartheta)\| \leq \int_{0}^{\vartheta}\left\|\Phi(s) B B^{*} \Phi^{*}(s)\right\| d s \leq \int_{0}^{\vartheta}\|\Phi(s)\| \cdot\|B\| \cdot\left\|B^{*}\right\| \cdot\left\|\Phi^{*}(s)\right\| d s \leq \vartheta e^{2 a \vartheta} b^{2} .
$$

For $\vartheta>0$, let us consider the operator $Q_{0}(\vartheta): \mathfrak{X} \rightarrow \mathfrak{X}$ given by:

$$
Q_{0}(\vartheta) x=\int_{0}^{\vartheta} \Phi(-t) B B^{*} \Phi^{*}(-t) x d t .
$$

We have $Q_{0}(\vartheta) \in \mathcal{L}(\mathfrak{X}, \mathfrak{X}), Q_{0}^{*}(\vartheta)=Q_{0}(\vartheta)$, and $Q_{0}(\vartheta) \geq 0$. By (23), we have $Q(\vartheta)=$ $\Phi(\vartheta) Q_{0}(\vartheta) \Phi^{*}(\vartheta)$.

Lemma 7. $\left\|Q_{0}(\vartheta)\right\| \leq \vartheta e^{2 a \vartheta} b^{2}$.

The proof of Lemma 7 is similar to the proof of Lemma 6 .

By ([49], Theorem 4.1.7), system (21) is exactly controllable on $[0, \vartheta]$ if and only if for some $\gamma>0$ and all $x \in \mathfrak{X}$ :

$$
\langle Q(\vartheta) x, x\rangle \geq \gamma\|x\|^{2} .
$$

Inequality (27) means that $Q(\vartheta) \geq \gamma I$. 
Lemma 8. System (21) is exactly controllable on $[0, \vartheta]$ if and only if, for some $\gamma_{1}>0$,

$$
Q_{0}(\vartheta) \geq \gamma_{1} I
$$

Proof. By (23),

$$
\begin{gathered}
\langle Q(\vartheta) x, x\rangle=\int_{0}^{\vartheta}\left\langle\Phi(\vartheta) \Phi(-t) B B^{*} \Phi^{*}(-t) \Phi^{*}(\vartheta) x, x\right\rangle d t=\int_{0}^{\vartheta}\left\|B^{*} \Phi^{*}(-t) \Phi^{*}(\vartheta) x\right\|^{2} d t \\
=\left|\Phi^{*}(\vartheta) x=y\right|=\int_{0}^{\vartheta}\left\|B^{*} \Phi^{*}(-t) y\right\|^{2} d t=\left\langle Q_{0}(\vartheta) y, y\right\rangle .
\end{gathered}
$$

$(\Longrightarrow)$. Suppose that system (21) is exactly controllable on $[0, \vartheta]$. Hence, for some $\gamma>0$ and all $x \in \mathfrak{X},(27)$ holds. Set $\gamma_{1}:=\gamma e^{-2 a \vartheta}$. Let $y \in \mathfrak{X}$ be an arbitrary element. Set $x:=\left(\Phi^{*}(\vartheta)\right)^{-1} y$. Then $y=\Phi^{*}(\vartheta) x$. Hence, $\|y\| \leq\left\|\Phi^{*}(\vartheta)\right\| \cdot\|x\| \leq e^{a \vartheta}\|x\|$. Therefore, $\|x\| \geq e^{-a \vartheta}\|y\|$. By using (29) and (27), we obtain:

$$
\left\langle Q_{0}(\vartheta) y, y\right\rangle=\langle Q(\vartheta) x, x\rangle \geq \gamma\|x\|^{2} \geq \gamma e^{-2 a \vartheta}\|y\|^{2}=\gamma_{1}\|y\|^{2}
$$

Hence, (28) holds.

$(\Longleftarrow)$. Suppose that $(28)$ holds. Set $\gamma:=\gamma_{1} e^{-2 a \vartheta}$. Let $x \in \mathfrak{X}$ be an arbitrary element. Set $y:=$ $\Phi^{*}(\vartheta) x$. Then $x=\left(\Phi^{*}(\vartheta)\right)^{-1} y$. Hence, $\|x\| \leq\left\|\left(\Phi^{*}(\vartheta)\right)^{-1}\right\| \cdot\|y\| \leq e^{a \vartheta}\|y\|$. Therefore, $\|y\| \geq e^{-a \vartheta}\|x\|$. By using (29) and (28), we obtain:

$$
\langle Q(\vartheta) x, x\rangle=\left\langle Q_{0}(\vartheta) y, y\right\rangle \geq \gamma_{1}\|y\|^{2} \geq \gamma_{1} e^{-2 a \vartheta}\|x\|^{2}=\gamma\|x\|^{2}
$$

Hence, (27) holds. Thus, system (21) is exactly controllable on $[0, \vartheta]$.

Consider the operator control system:

$$
\dot{Y}(t)=A Y(t)+B U_{1}(t)
$$

where $Y(t): \mathfrak{X} \rightarrow \mathfrak{X}, U_{1}(t): \mathfrak{X} \rightarrow \mathfrak{U}, t \in \mathbb{R}$.

Lemma 9. Let system (21) be exactly controllable on $[0, \vartheta]$ for some $\vartheta>0$. Then there exists $\sigma(=2 \vartheta)>0$ such that for an arbitrary $\lambda \in \mathbb{R}$ there exists a continuous operator control function $[0, \sigma] \ni t \mapsto U_{1}(t) \in \mathcal{L}(\mathfrak{X}, \mathfrak{U})$ such that $\left\|U_{1}(t)\right\| \leq \alpha_{1}$ for some $\alpha_{1} \geq 0$ for all $t \in[0, \sigma]$, steering the solution of (30) from:

$$
Y(0)=I
$$

to

$$
Y(\sigma)=e^{\lambda \sigma} \Phi(\sigma)
$$

so that the operator solution $Y(t)$ of $(30)$ is a linear bounded operator function with a bounded inverse:

$$
\|Y(t)\| \leq \beta_{1}, \quad\left\|Y^{-1}(t)\right\| \leq \beta_{2}, \quad t \in[0, \sigma]
$$

Proof. Let system (21) be exactly controllable on $[0, \vartheta], \vartheta>0$. Set $\sigma:=2 \vartheta$. Suppose that $\lambda \in \mathbb{R}$ is given. A solution of (30) with the initial condition (31) has the form:

$$
Y(t)=\Phi(t) \cdot\left(I+\int_{0}^{t} \Phi(-s) B U_{1}(s) d s\right)
$$


Condition (32) holds if and only if:

$$
I+\int_{0}^{\sigma} \Phi(-s) B U_{1}(s) d s=e^{\lambda \sigma} I .
$$

We will search for $U_{1}(t)$ in the form:

$$
U_{1}(t)=B^{*} \Phi^{*}(-t) H,
$$

where $H \in \mathcal{L}(\mathfrak{X}, \mathfrak{X})$. Then, it follows from (35) that:

$$
I+Q_{0}(\sigma) H=e^{\lambda \sigma} I
$$

By definition (26) of $Q_{0}(\cdot)$, we have $Q_{0}(\sigma) \geq Q_{0}(\vartheta)$. By Lemma $8, Q_{0}(\vartheta) \geq \gamma_{1} I$ for some $\gamma_{1}>0$. Hence, $Q_{0}^{-1}(\sigma) \in \mathcal{L}(\mathfrak{X}, \mathfrak{X})$ and $\left\|Q_{0}^{-1}(\sigma)\right\| \leq \delta_{1}$ for some $\delta_{1}>0$. Finding $H$ from (37), we obtain:

$$
H=\left(e^{\lambda \sigma}-1\right) Q_{0}^{-1}(\sigma) .
$$

Substituting (38) in (36), we obtain:

$$
U_{1}(t)=B^{*} \Phi^{*}(-t) Q_{0}^{-1}(\sigma)\left(e^{\lambda \sigma}-1\right), \quad t \in[0, \sigma] .
$$

We have,

$$
\left\|U_{1}(t)\right\| \leq\left\|B^{*}\right\| \cdot\left\|\Phi^{*}(-t)\right\| \cdot\left\|Q_{0}^{-1}(\sigma)\right\| \cdot\left|e^{\lambda \sigma}-1\right| \leq b e^{a \sigma} \delta_{1}\left|e^{\lambda \sigma}-1\right|=: \alpha_{1}, \quad t \in[0, \sigma] .
$$

Substituting (39) in (34), we obtain:

$$
Y(t)=\Phi(t) R(t)
$$

where

$$
R(t)=I+\int_{0}^{t} \Phi(-s) B B^{*} \Phi^{*}(-s) d s Q_{0}^{-1}(\sigma)\left(e^{\lambda \sigma}-1\right) .
$$

We have, for all $t \in[0, \sigma]$,

$$
\|R(t)\| \leq\|I\|+\int_{0}^{t}\left\|\Phi(-s) B B^{*} \Phi^{*}(-s)\right\| d s \cdot\left\|Q_{0}^{-1}(\sigma)\right\| \cdot\left|e^{\lambda \sigma}-1\right| \leq 1+\sigma e^{2 a \sigma} b^{2} \delta_{1}\left|e^{\lambda \sigma}-1\right|,
$$

hence,

$$
\|Y(t)\| \leq\|\Phi(t)\| \cdot\|R(t)\| \leq e^{a \sigma}\left(1+\sigma e^{2 a \sigma} b^{2} \delta_{1}\left|e^{\lambda \sigma}-1\right|\right)=: \beta_{1}, \quad t \in[0, \sigma] .
$$

Thus, the first inequality in (33) holds.

Let us show that $R(t)$ has a bounded inverse for all $t \in[0, \sigma]$. Consider the operator

$$
P(t):=R(t) Q_{0}(\sigma)=Q_{0}(\sigma)+\left(e^{\lambda \sigma}-1\right) \int_{0}^{t} \Phi(-s) B B^{*} \Phi^{*}(-s) d s .
$$

We have $P^{*}(t)=P(t), t \in[0, \sigma]$, and

$$
\begin{aligned}
P(t)= & \int_{0}^{\sigma} \Phi(-s) B B^{*} \Phi^{*}(-s) d s-\int_{0}^{t} \Phi(-s) B B^{*} \Phi^{*}(-s) d s+e^{\lambda \sigma} \int_{0}^{t} \Phi(-s) B B^{*} \Phi^{*}(-s) d s \\
& =\int_{t}^{\sigma} \Phi(-s) B B^{*} \Phi^{*}(-s) d s+e^{\lambda \sigma} \int_{0}^{t} \Phi(-s) B B^{*} \Phi^{*}(-s) d s=: P_{1}(t)+P_{2}(t) .
\end{aligned}
$$

We see that $P_{i}^{*}(t)=P_{i}(t)$ and $P_{i}(t) \geq 0, i=1,2, t \in[0, \sigma]$. 
Let $t \in[0, \vartheta]$. Then,

$$
\begin{gathered}
P_{1}(t)=\int_{t}^{\sigma} \Phi(-s) B B^{*} \Phi^{*}(-s) d s \\
=\int_{t}^{\vartheta} \Phi(-s) B B^{*} \Phi^{*}(-s) d s+\int_{\vartheta}^{2 \vartheta} \Phi(-s) B B^{*} \Phi^{*}(-s) d s=: P_{3}(t)+P_{4}(\vartheta) .
\end{gathered}
$$

We have $P_{3}^{*}(t)=P_{3}(t), P_{4}^{*}(\vartheta)=P_{4}(\vartheta)$,

$$
P_{3}(t)=\int_{t}^{\vartheta} \Phi(-s) B B^{*} \Phi^{*}(-s) d s \geq 0 .
$$

Next,

$$
\begin{aligned}
P_{4}(\vartheta) & =\int_{\vartheta}^{2 \vartheta} \Phi(-s) B B^{*} \Phi^{*}(-s) d s=\Phi^{-1}(\vartheta) \int_{\vartheta}^{2 \vartheta} \Phi(\vartheta-s) B B^{*} \Phi^{*}(\vartheta-s) d s\left(\Phi^{*}(\vartheta)\right)^{-1} \\
& =\Phi^{-1}(\vartheta) \int_{0}^{\vartheta} \Phi(-t) B B^{*} \Phi^{*}(-t) d t\left(\Phi^{*}(\vartheta)\right)^{-1}=\Phi^{-1}(\vartheta) Q_{0}(\vartheta)\left(\Phi^{*}(\vartheta)\right)^{-1} .
\end{aligned}
$$

Since system (21) is exactly controllable on $[0, \vartheta]$, we have, by Lemma $8, Q_{0}(\vartheta) \geq \gamma_{1} I$. Therefore, $P_{4}(\vartheta) \geq \gamma_{2} I$ for some $\gamma_{2} \geq 0$ (namely, for $\gamma_{2}:=\gamma_{1} e^{-2 a \vartheta}$; the proof is similar to the proof of Lemma 8). So, we have $P_{1}(t)=P_{3}(t)+P_{4}(\vartheta) \geq \gamma_{2} I$ for $t \in[0, \vartheta]$. Thus, $P(t)=P_{1}(t)+P_{2}(t) \geq \gamma_{2} I$ for $t \in[0, \vartheta]$.

Let $t \in[\vartheta, 2 \vartheta]$. We have,

$$
P_{2}(t)=e^{\lambda \sigma} \int_{0}^{t} \Phi(-s) B B^{*} \Phi^{*}(-s) d s \geq e^{\lambda \sigma} \int_{0}^{\vartheta} \Phi(-s) B B^{*} \Phi^{*}(-s) d s=e^{\lambda \sigma} Q_{0}(\vartheta) \geq e^{\lambda \sigma} \gamma_{1} I .
$$

So, $P(t)=P_{1}(t)+P_{2}(t) \geq e^{\lambda \sigma} \gamma_{1} I$ for $t \in[\vartheta, 2 \vartheta]$.

Thus, for all $t \in[0, \sigma]$, we have $P(t) \geq \gamma_{3} I>0$ where $\gamma_{3}:=\min \left\{\gamma_{2}, \gamma_{1} e^{\lambda \sigma}\right\}$. Hence, there exists an inverse $P^{-1}(t) \in \mathcal{L}(\mathfrak{X}, \mathfrak{X})$ and $\left\|P^{-1}(t)\right\| \leq \delta_{2}$ for some $\delta_{2}>0$ for all $t \in[0, \sigma]$. Then $R(t)=P(t) Q_{0}^{-1}(\sigma)$ has a bounded inverse:

$$
R^{-1}(t)=Q_{0}(\sigma) P^{-1}(t)
$$

and, by using the estimation in Lemma 7, we obtain:

$$
\left\|R^{-1}(t)\right\| \leq\left\|Q_{0}(\sigma)\right\| \cdot\left\|P^{-1}(t)\right\| \leq \sigma e^{2 a \sigma} b^{2} \delta_{2}=: \delta_{3} .
$$

By (40), we obtain:

$$
Y^{-1}(t)=R^{-1}(t) \Phi^{-1}(t), \quad t \in[0, \sigma],
$$

and

$$
\left\|Y^{-1}(t)\right\| \leq\left\|R^{-1}(t)\right\| \cdot\left\|\Phi^{-1}(t)\right\| \leq \delta_{3} e^{a \sigma}=: \beta_{2}, \quad t \in[0, \sigma] .
$$

Theorem 2. Let system (21) be exactly controllable on $[0, \vartheta]$ for some $\vartheta>0$. Then system (21) admits a $\lambda$-transformation.

Proof. Let system (21) be exactly controllable on $[0, \vartheta], \vartheta>0$. Set: $\sigma:=2 \vartheta$. Suppose that $\lambda \in \mathbb{R}$ is given. Let us construct the control function $U_{1}(t), t \in[0, \sigma]$, in accordance with Lemma 9 . Set

$$
U_{2}(t):=U_{1}(t) Y^{-1}(t), \quad t \in[0, \sigma] .
$$

Then $U_{2}(t) \in \mathcal{L}(\mathfrak{X}, \mathfrak{U}), t \in[0, \sigma]$, and

$$
\left\|U_{2}(t)\right\| \leq\left\|U_{1}(t)\right\| \cdot\left\|Y^{-1}(t)\right\| \leq \alpha_{1} \beta_{2}, \quad t \in[0, \sigma] .
$$


We have

$$
U_{1}(t)=U_{2}(t) Y(t), \quad t \in[0, \sigma]
$$

Let us substitute (43) in (30). Then we obtain that the function $Y(t), t \in[0, \sigma]$, defined by $(40)$ is a solution of the system:

$$
\dot{Y}(t)=\left(A+B U_{2}(t)\right) Y(t)
$$

satisfying the initial condition (31). Hence,

$$
Y(t)=\Phi_{U_{2}}(t, 0), \quad t \in[0, \sigma]
$$

where $\Phi_{U_{2}}(t, s), t, s \in[0, \sigma]$, is the evolution operator of system (44). It follows from (45) and (32) that:

$$
\Phi_{U_{2}}(\sigma, 0)=e^{\lambda \sigma} \Phi(\sigma) \text {. }
$$

Let us extend the function $U_{2}(t), t \in[0, \sigma]$, onto $\mathbb{R}$ periodically with the period $\sigma$, i.e., construct the function:

$$
U(t) \equiv U_{2}(t-k \sigma), \quad t \in[k \sigma,(k+1) \sigma), \quad k \in \mathbb{Z}
$$

Consider the system:

$$
\dot{Y}(t)=(A+B U(t)) Y(t), \quad t \in \mathbb{R} .
$$

System (48) is $\sigma$-periodic. Therefore, the evolution operator $\Phi_{U}(t, s)$ of system (48) satisfies the following condition:

$$
\Phi_{U}(t+k \sigma, s+k \sigma)=\Phi_{U}(t, s)
$$

for all $t, s \in \mathbb{R}$ and $k \in \mathbb{Z}$. In particular,

$$
\Phi_{U}((k+1) \sigma, k \sigma)=\Phi_{U}(\sigma, 0)=\Phi_{U_{2}}(\sigma, 0) .
$$

From (49), (46), and the equality $\Phi(\sigma)=\Phi((k+1) \sigma, k \sigma)$, it follows that:

$$
\Phi_{U}((k+1) \sigma, k \sigma)=e^{\lambda \sigma} \Phi((k+1) \sigma, k \sigma) .
$$

Thus, the required equality is proved. The function $U(\cdot)$ is piecewise continuous and:

$$
\sup _{t \in \mathbb{R}}\|U(t)\|=\sup _{t \in[0, \sigma)}\left\|U_{2}(t)\right\| \leq \alpha_{1} \beta_{2}
$$

The theorem is proved.

Corollary 1. Let system (21) be exactly controllable on $[0, \vartheta]$ for some $\vartheta>0$. Then, for any $\lambda \in \mathbb{R}$, there exists an admissible gain operator function $U(\cdot)$ such that the closed-loop system:

$$
\dot{x}(t)=(A+B U(t)) x(t)
$$

and system (7) are kinematically similar on $\mathbb{R}$.

Corollary 1 follows from Theorems 1 and 2.

Theorem 3. Let system (21) be exactly controllable on $[0, \vartheta]$ for some $\vartheta>0$. Then the upper Bohl exponent of system (21) is arbitrarily assignable by the linear state feedback (18).

Proof. Let $\mu \in \mathbb{R}$ be given. Set,

$$
\lambda:=\mu-\varkappa(A) .
$$


For this $\lambda$, let us construct, by Corollary 1 , an admissible gain operator function $U(\cdot)$ such that system (51) and system (7) are kinematically similar on $\mathbb{R}$. By Lemma 4, we have:

$$
\varkappa(A+B U)=\varkappa(A+\lambda I) .
$$

By Lemma 2, we have:

$$
\varkappa(A+\lambda I)=\varkappa(A)+\lambda .
$$

From (53), (54), and (52), it follows that $\varkappa(A+B U)=\mu$ as required.

\section{Example}

Let $\mathfrak{X}=\mathfrak{U}=\ell_{2}$ where $\ell_{2}$ is the is the space of all sequences $x=\left(x_{1}, x_{2}, \ldots, x_{n}, \ldots\right)$ with the norm $\|x\|=\left(\sum_{i=1}^{\infty}\left|x_{i}\right|^{2}\right)^{1 / 2}$. The space $\ell_{2}$ is a separable Hilbert space ([51], §56). Consider a linear control system:

$$
\dot{x}(t)=A x(t)+B u(t), \quad t \in \mathbb{R}, \quad x \in \mathfrak{X}, \quad u \in \mathfrak{U},
$$

where

$$
\begin{aligned}
& A:\left(x_{1}, x_{2}, x_{3}, x_{4}, \ldots\right) \mapsto\left(-x_{2}, x_{1},-x_{4}, x_{3}, \ldots\right), \\
& B:\left(x_{1}, x_{2}, x_{3}, x_{4}, \ldots\right) \mapsto\left(x_{1}, 0, x_{2}, 0, x_{3}, 0, \ldots\right) .
\end{aligned}
$$

Considering elements of $\ell_{2}$ as column-vectors with an infinite number of coordinates, one can identify the operators $A$ and $B$ with the following matrices with an infinite number of rows and columns:

$$
A=\left[\begin{array}{ccccc}
0 & -1 & 0 & 0 & \ldots \\
1 & 0 & 0 & 0 & \ldots \\
0 & 0 & 0 & -1 & \ldots \\
0 & 0 & 1 & 0 & \ldots \\
\vdots & \vdots & \vdots & \vdots & \ddots
\end{array}\right], \quad B=\left[\begin{array}{cccc}
1 & 0 & 0 & \ldots \\
0 & 0 & 0 & \ldots \\
0 & 1 & 0 & \ldots \\
0 & 0 & 0 & \ldots \\
\vdots & \vdots & \vdots & \ddots
\end{array}\right]
$$

block-diagonal form:

Consider the matrices $F=\left[\begin{array}{cc}0 & -1 \\ 1 & 0\end{array}\right], G=\left[\begin{array}{l}1 \\ 0\end{array}\right]$. One can write the matrices (58) in the following

$$
A=\operatorname{diag}\{F, F, \ldots, F, \ldots\}, \quad B=\operatorname{diag}\{G, G, \ldots, G, \ldots\} .
$$

We will use the following denotations for the matrices of the form (59):

$$
A=\operatorname{diag}[F]_{\infty}, \quad B=\operatorname{diag}[G]_{\infty} .
$$

Set $G_{1}:=F G$. Then $G_{1}=\left[\begin{array}{l}0 \\ 1\end{array}\right]$. Hence, $A B=\operatorname{diag}\left[G_{1}\right]_{\infty}$. Therefore, $\operatorname{span}\{B \mathfrak{U}, A B \mathfrak{U}\}=\mathfrak{X}$. It follows that system (55), (56), (57) is exactly controllable on $[0, \vartheta]$ for any $\vartheta>0$. Let us take $\vartheta=\pi$. Let us show that the upper Bohl exponent of system (55), (56), (57) is arbitrarily assignable by linear state feedback (18).

Consider the system:

$$
\dot{y}(t)=F y(t), \quad t \in \mathbb{R}, \quad y \in \mathbb{R}^{2} .
$$


The evolution operator $\Gamma(t, s)$ of system (60) has the form $\Gamma(t, s)=\Gamma(t-s)$ where $\Gamma(t)=\exp (F t)$. Calculating the matrix exponent, we obtain that:

$$
\Gamma(t)=\left[\begin{array}{cc}
\cos t & -\sin t \\
\sin t & \cos t
\end{array}\right] .
$$

Let us construct the evolution operator $\Phi(t, s)$ of the free system:

$$
\dot{x}(t)=A x(t), \quad t \in \mathbb{R} .
$$

We obtain $\Phi(t, s)=\Phi(t-s)$ where $\Phi(t)=\operatorname{diag}[\Gamma(t)]_{\infty}$. Hence, $\Phi(\tau+s, \tau)=\operatorname{diag}[\Gamma(s)]_{\infty}$. For any $y=\operatorname{col}\left(y_{1}, y_{2}\right) \in \mathbb{R}^{2}$ we have:

$$
\|\Gamma(s) y\|^{2}=\left(y_{1} \cos s-y_{2} \sin s\right)^{2}+\left(y_{1} \sin s+y_{2} \cos s\right)^{2}=y_{1}^{2}+y_{2}^{2}=\|y\|^{2} .
$$

From this, it follows that $\|\Phi(\tau+s, \tau) x\|^{2}=\|x\|^{2}$ for all $\tau, s \in \mathbb{R}$ and $x \in \mathfrak{X}$. Hence, $\| \Phi(\tau+$ $s, \tau) \|=1$. So, $\ln \|\Phi(\tau+s, \tau)\|=0$. Thus, $\varkappa(A)=0$.

Let an arbitrary $\mu \in \mathbb{R}$ be given. Set $\lambda:=\mu-\varkappa(A)=\mu$. Let us construct $U(\cdot)$, by Theorem 2, that ensures equality (50), and, hence, by Corollary 1 , kinematic similarity of systems (51) and (7), and, thus, the equality $\varkappa(A+B U)=\lambda=\mu$. Set $\sigma:=2 \vartheta=2 \pi$. We have:

$$
\Phi(\sigma)=\Phi(2 \pi)=\operatorname{diag}[\Gamma(2 \pi)]_{\infty}=\operatorname{diag}\left[I_{2}\right]_{\infty}
$$

where $I_{2}=\left[\begin{array}{ll}1 & 0 \\ 0 & 1\end{array}\right]$, i.e., $\Phi(\sigma)=I \in \mathcal{L}(\mathfrak{X}, \mathfrak{X})$. Next,

$$
\Gamma(-s) G G^{*} \Gamma^{*}(-s)=\left[\begin{array}{cc}
\cos ^{2} s & -\cos s \sin s \\
-\cos s \sin s & \sin ^{2} s
\end{array}\right]
$$

Set $\Sigma(t):=\int_{0}^{t} \Gamma(-s) G G^{*} \Gamma^{*}(-s) d s$. Then,

$$
\Sigma(t)=\left[\begin{array}{ll}
\frac{t}{2}+\frac{\sin 2 t}{\cos 2 t-1} & \frac{\cos 2 t-1}{4} \\
\frac{t}{2}-\frac{4 \sin 2 t}{4}
\end{array}\right],
$$

and, hence, $\Sigma(\sigma)=\Sigma(2 \pi)=\pi I_{2}$. We have:

$$
\begin{aligned}
Q_{0}(\sigma)= & \int_{0}^{\sigma} \Phi(-s) B B^{*} \Phi^{*}(-s) d s=\int_{0}^{\sigma} \operatorname{diag}[\Gamma(-s)]_{\infty} \operatorname{diag}\left[G G^{*}\right]_{\infty} \operatorname{diag}\left[\Gamma^{*}(s)\right]_{\infty} d s \\
& =\int_{0}^{\sigma} \operatorname{diag}\left[\Gamma(-s) G G^{*} \Gamma^{*}(s)\right]_{\infty} d s=\operatorname{diag}[\Sigma(\sigma)]_{\infty}=\pi I \in \mathcal{L}(\mathfrak{X}, \mathfrak{X}) .
\end{aligned}
$$

So, $Q_{0}^{-1}(\sigma)=\frac{1}{\pi} I \in \mathcal{L}(\mathfrak{X}, \mathfrak{X})$, hence, by (38), $H=\frac{e^{2 \pi \lambda}-1}{\pi} I \in \mathcal{L}(\mathfrak{X}, \mathfrak{X})$. Denote,

$$
\alpha:=\frac{e^{2 \pi \lambda}-1}{2 \pi} .
$$

By using (39), we obtain,

$$
U_{1}(t)=\operatorname{diag}\left[V_{1}(t)\right]_{\infty}, \quad \text { where } \quad V_{1}(t)=[2 \alpha \cos t,-2 \alpha \sin t] .
$$


Next, by (41), we have $R(t)=\operatorname{diag}[K(t)]_{\infty}$, where $K(t)=I_{2}+2 \alpha \Sigma(t)$. Set $S(t):=\Gamma(t) K(t)$. Multiplying $\Gamma(t)$ by $K(t)$, we obtain that:

$$
S(t)=\left[\begin{array}{cc}
(1+\alpha t) \cos t+\alpha \sin t & -(1+\alpha t) \sin t \\
(1+\alpha t) \sin t & (1+\alpha t) \cos t-\alpha \sin t
\end{array}\right] .
$$

By (40), we have,

$$
Y(t)=\Phi(t) R(t)=\operatorname{diag}[\Gamma(t)]_{\infty} \operatorname{diag}[K(t)]_{\infty}=\operatorname{diag}[\Gamma(t) K(t)]_{\infty}=\operatorname{diag}[S(t)]_{\infty} .
$$

Finding $\Delta(t):=\operatorname{det} S(t)$ from (63), we obtain that:

$$
\Delta(t)=(1+\alpha t)^{2}-\alpha^{2} \sin ^{2} t .
$$

It is easy to check that, for all $t \in[0, \sigma]: \Delta^{\prime}(t)>0$, if $\alpha>0 ; \Delta^{\prime}(t)<0$, if $\alpha<0$; and $\Delta^{\prime}(t)=0$, if $\alpha=0$. Hence, for all $t \in[0, \sigma]$ : if $\alpha>0$, then $\Delta(t) \geq \Delta(0)=1$; if $\alpha<0$, then $\Delta(t) \geq \Delta(2 \pi)=e^{4 \pi \lambda}>$ 0 ; if $\alpha=0$, then $\Delta(t)=1$. Thus, $\Delta(t)$ is separated from zero.

From (63), we obtain that

$$
S^{-1}(t)=\frac{1}{\Delta(t)}\left[\begin{array}{cc}
(1+\alpha t) \cos t-\alpha \sin t & (1+\alpha t) \sin t \\
-(1+\alpha t) \sin t & (1+\alpha t) \cos t+\alpha \sin t
\end{array}\right] .
$$

By (64), we have:

$$
Y^{-1}(t)=\operatorname{diag}\left[S^{-1}(t)\right]_{\infty}
$$

Constructing $U_{2}(t)$ according to (42), by using (62), (66), and (65), we obtain:

$$
U_{2}(t)=\operatorname{diag}\left[V_{2}(t)\right]_{\infty}, \quad V_{2}(t)=\frac{1}{\Delta(t)}\left[2 \alpha(1+\alpha t)-2 \alpha^{2} \sin t \cos t,-2 \alpha^{2} \sin ^{2} t\right] .
$$

From (67) we obtain:

$$
F+G V_{2}(t)=\left[\begin{array}{cc}
\frac{2 \alpha(1+\alpha t)-2 \alpha^{2} \sin t \cos t}{\Delta(t)} & \frac{-(1+\alpha t)^{2}-\alpha^{2} \sin ^{2} t}{\Delta(t)} \\
1 & 0
\end{array}\right]
$$

One can check that the matrix (63) satisfies the following matrix differential equation:

$$
\dot{S}(t)=\left(F+G V_{2}(t)\right) S(t), \quad t \in[0, \sigma] .
$$

Next, by (67), we have:

$$
A+B U_{2}(t)=\operatorname{diag}\left[F+G V_{2}(t)\right]_{\infty} .
$$

Due to (68) and (69), the function (64) satisfies the system:

$$
\dot{Y}(t)=\left(A+B U_{2}(t)\right) Y(t), \quad t \in[0, \sigma],
$$

and $Y(0)=I$. Hence, (45) and (46) holds. Constructing $U(t)$ according to (47), we obtain:

$$
\begin{aligned}
U(t)=\operatorname{diag}[V(t)]_{\infty}, \quad V(t)= & \frac{1}{\Delta(t-2 \pi k)}\left[2 \alpha(1+\alpha(t-2 \pi k))-2 \alpha^{2} \sin t \cos t,-2 \alpha^{2} \sin ^{2} t\right], \\
& t \in[2 \pi k, 2 \pi(k+1)), \quad k \in \mathbb{Z} .
\end{aligned}
$$


By Theorem 2, the gain operator function (70) with $\alpha$ defined by (61) ensures equality (50), kinematic similarity of systems:

$$
\dot{x}(t)=(A+B U(t)) x(t), \quad t \in \mathbb{R},
$$

and

$$
\dot{x}(t)=(A+\lambda I) x(t), \quad t \in \mathbb{R},
$$

on $\mathbb{R}$, and the equality $\varkappa(A+B U)=\lambda=\mu$.

For numerical simulation, let us construct the projection of systems (71) and (72) into the space $\mathbb{R}^{2}=\left\{\left(x_{1}, x_{2}\right), x_{1}, x_{2} \in \mathbb{R}\right\}$. We obtain the systems

$$
\dot{y}(t)=(F+G V(t)) y(t), \quad t \in \mathbb{R}, \quad y \in \mathbb{R}^{2},
$$

and

$$
\dot{y}(t)=(F+\lambda E) y(t), \quad t \in \mathbb{R}, \quad y \in \mathbb{R}^{2} .
$$

Here $E$ is the identity $(2 \times 2)$-matrix. Systems (73) and (74) are kinematically similar, hence, since $\varkappa(F)=0$, we have $\varkappa(F+G V)=\varkappa(F+\lambda E)=\lambda$. Let us take, for example, $\lambda=-1 / 4$. The equality $\varkappa(F+\lambda E)=-1 / 4$ means that system (74) (and (73)) is uniformly exponentially stable with the decay rate $1 / 4$.

Let $\Xi(t, s)$ denote the evolution matrix of system (73) and $\Omega(t, s)$ denote the evolution matrix of system (74). Let,

$$
\Xi(t, 0)=:\left[\begin{array}{ll}
\xi_{11}(t) & \xi_{12}(t) \\
\xi_{21}(t) & \xi_{22}(t)
\end{array}\right], \quad \Omega(t, 0)=:\left[\begin{array}{ll}
\omega_{11}(t) & \omega_{12}(t) \\
\omega_{21}(t) & \omega_{22}(t)
\end{array}\right]
$$

We have $\left.\Xi(t, 0)\right|_{t=0}=\left.\Omega(t, 0)\right|_{t=0}=E$. Let us construct the graphs of the functions $\xi_{i j}(t), \omega_{i j}(t)$ (see, Figures 1-4).

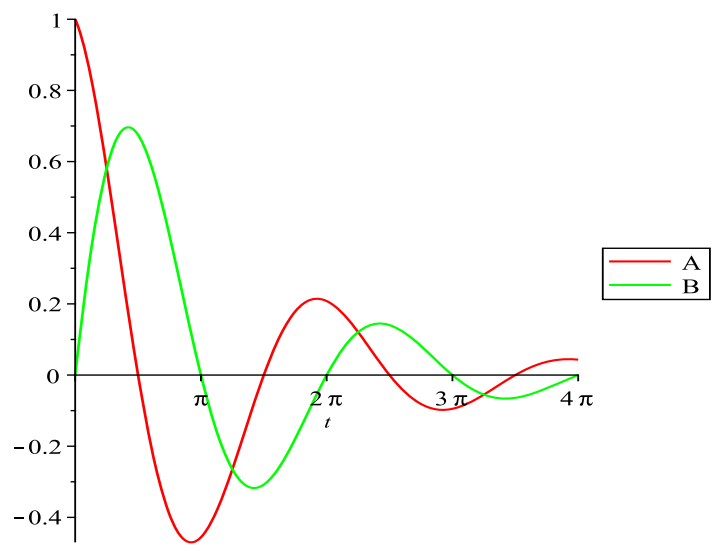

Figure 1. Graphs of the functions $A=\omega_{11}(t), B=\omega_{21}(t)$. 


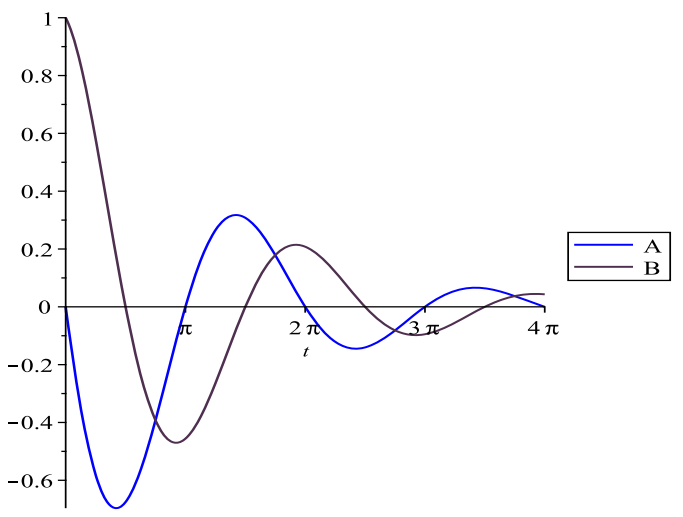

Figure 2. Graphs of the functions $A=\omega_{12}(t), B=\omega_{22}(t)$.

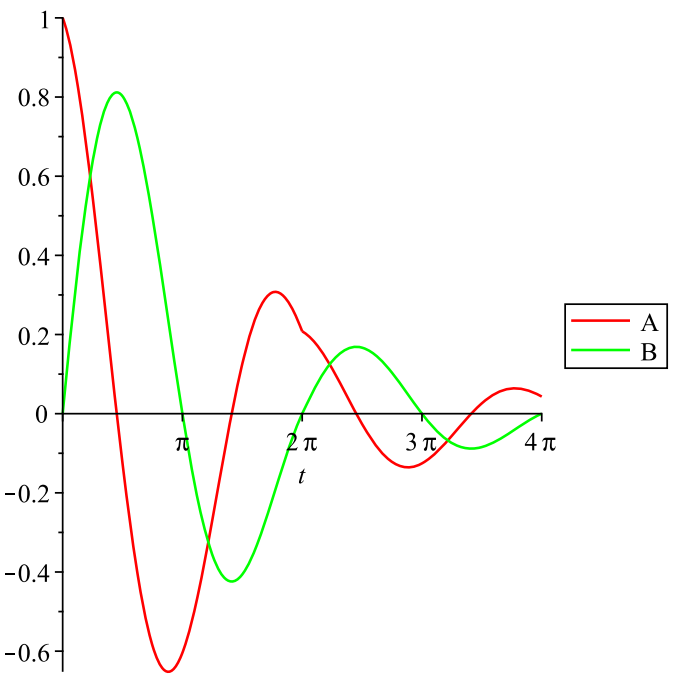

Figure 3. Graphs of the functions $A=\xi_{11}(t), B=\xi_{21}(t)$.

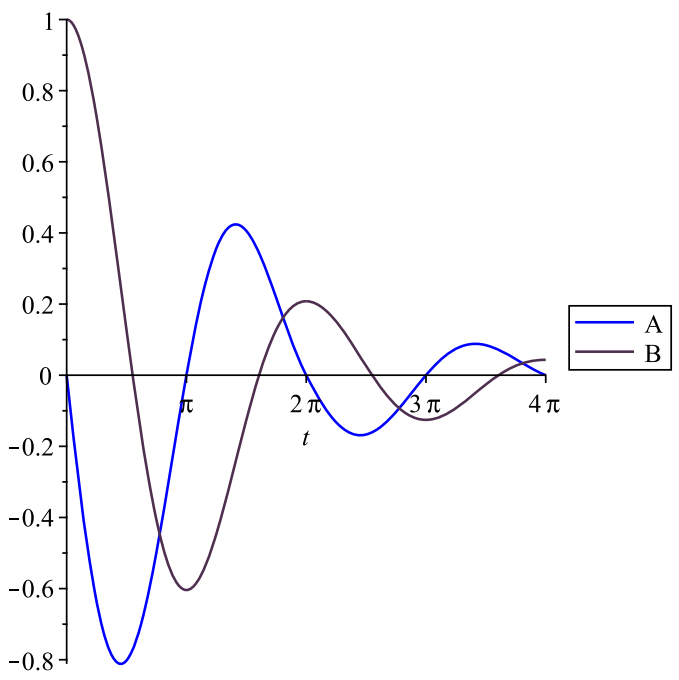

Figure 4. Graphs of the functions $A=\xi_{12}(t), B=\xi_{22}(t)$. 
One can see from system (73) and (74) (and from the graphs) that:

$$
\begin{array}{ll}
\omega_{11}(2 \pi)=\xi_{11}(2 \pi)=e^{-\pi / 2} \approx 0.2079, & \omega_{12}(2 \pi)=\xi_{12}(2 \pi)=0, \\
\omega_{21}(2 \pi)=\xi_{21}(2 \pi)=0, & \omega_{22}(2 \pi)=\xi_{11}(2 \pi)=e^{-\pi / 2} \approx 0.2079 .
\end{array}
$$

One can see also that the functions $\omega_{i j}$ are smooth. Since the matrix of system (73) is piecewise continuous, the matrix function $\Xi(t, 0)$ is piecewise smooth and its derivative can be discontinuous at the points $t=2 \pi k$. Calculating the one-sided limits from (73), we obtain that:

$$
\begin{gathered}
\left.\dot{\xi}_{11}(t)\right|_{t=2 \pi-0}=\left(e^{-\pi / 2}-1\right) / \pi \approx-0.2521,\left.\quad \dot{\xi}_{11}(t)\right|_{t=2 \pi+0}=\left(e^{-\pi / 2}-1\right) e^{-\pi / 2} / \pi \approx-0.0524, \\
\left.\dot{\xi}_{12}(t)\right|_{t=2 \pi-0}=\left.\dot{\xi}_{12}(t)\right|_{t=2 \pi+0}=-e^{-\pi / 2},\left.\quad \dot{\xi}_{21}(t)\right|_{t=2 \pi-0}=\left.\dot{\xi}_{21}(t)\right|_{t=2 \pi+0}=e^{-\pi / 2}, \\
\left.\dot{\xi}_{22}(t)\right|_{t=2 \pi-0}=\left.\dot{\xi}_{22}(t)\right|_{t=2 \pi+0}=0,
\end{gathered}
$$

i.e., only the function $\xi_{11}(t)$ has the discontinuous derivative at the point $t=2 \pi$. This is confirmed by the graphs.

It follows from (75) that:

$$
\Xi(2 \pi, 0)=\Omega(2 \pi, 0)=\left[\begin{array}{cc}
e^{-\pi / 2} & 0 \\
0 & e^{-\pi / 2}
\end{array}\right] \approx\left[\begin{array}{cc}
0.2079 & 0 \\
0 & 0.2079
\end{array}\right]
$$

By periodicity, we have:

$$
\Xi(4 \pi, 0)=\Omega(4 \pi, 0)=\left[\begin{array}{cc}
e^{-\pi} & 0 \\
0 & e^{-\pi}
\end{array}\right] \approx\left[\begin{array}{cc}
0.0432 & 0 \\
0 & 0.0432
\end{array}\right],
$$

and so on, $\Xi(2 \pi k, 0)=\Omega(2 \pi k, 0)=e^{-\pi k / 2} E, k \in \mathbb{Z}$. The graphs confirm asymptotic equivalence of the behavior of solutions of systems (73) and (74).

Remark 2. The advantage of the developed method is that it allows us to establish the exact asymptotics (i.e., exact equality $\varkappa(A+B U)=\mu$ ) for the closed-loop system, in contrast to, e.g., [35], from which one can only obtain the inequality $\Lambda(A+B U) \leq \varkappa$ for the upper Lyapunov exponent $\Lambda$ of the closed-loop system. The problem of exact assignment of the upper Bohl exponent for a system in infinite-dimensional space in the presented formulation has not been previously investigated. Moreover, the developed method allows us to assign exact values for other asymptotic invariants of the closed-loop system (central exponents, exponential exponents etc.). A disadvantage is that the analytical expressions for the controller (and for solutions of the closed-loop system) can be complicated, in contrast to the stabilization problem [35]. This method can be applied to any system with the property of exact controllability. The choice of matrices in the example in a rather simple form was made for illustrative purposes because in this case the analytical expressions for the controller and for the solutions of the closed-loop system is not very complicated.

\section{Conclusions}

For a linear time-invariant control system in a Hilbert space with bounded operator coefficients, we examined the problem of arbitrary assignment of the upper Bohl exponent by means of linear state feedback with a time-varying linear bounded gain operator function. We have proved that the property of exact controllability of the open-loop system is sufficient for arbitrary assignability of the upper Bohl exponent of the closed-loop system. We plan to extend these results to systems without necessarily bounded operator $A$ but generating a $C_{0}$-continuous semigroup. We plan to prove similar results for systems with dynamic output feedback. Further development of these results may be their extension to systems with periodic coefficients and with arbitrary time-varying non-periodic 
coefficients, to systems in general Banach spaces, or to systems with discrete time. We expect to apply the results to specific systems, for example, to systems with delays, considering them as abstract systems of differential equations in an infinite-dimensional space.

Author Contributions: Conceptualization, V.Z.; methodology, V.Z.; formal analysis, V.Z. and M.Z.; investigation, V.Z. and M.Z.; writing-original draft preparation, V.Z. and M.Z.; writing-review and editing, V.Z. and M.Z.; visualization, V.Z. and M.Z.; supervision, V.Z.; project administration, V.Z.; funding acquisition, V.Z. All authors have read and agreed to the published version of the manuscript.

Funding: This research was funded by the Ministry of Science and Higher Education of the Russian Federation in the framework of state assignment No. 075-00232-20-01, Project 0827-2020-0010 “Development of the theory and methods of control and stabilization of dynamical systems".

Conflicts of Interest: The authors declare no conflict of interest.

\section{References}

1. Popov, V.M. Hyperstability and optimality of automatic systems with several control functions. Revue Roumaine des Sciences Techniques-Serie Electrotechnique et Energetique 1964, 9, 629-690.

2. Wonham, W.M. On pole assignment in multi-input controllable linear systems. IEEE Trans. Autom. Control. 1967, 12, 660-665. [CrossRef]

3. Brunovsky, P. Controllability and linear closed-loop controls in linear periodic systems. J. Differ. Equ. 1969, 6, 296-313. 10.1016/0022-0396(69)90019-9. [CrossRef]

4. Silverman, L.M. Transformation of time-variable systems to canonical (phase-variable) form. IEEE Trans. Autom. Control. 1966, 11, 300-303. [CrossRef]

5. Wolovich, W.A. On the stabilization of controllable system. IEEE Trans. Autom. Control. 1968, 13, 569-572. [CrossRef]

6. Silverman, L.M.; Anderson, B.D.O. Controllability, observability and stability of linear systems. SIAM J. Control 1968, 6, 121-130. [CrossRef]

7. Ikeda, M.; Maeda, H.; Kodama, S. Stabilization of linear systems. SIAM J. Control 1972, 10, 716-729. [CrossRef]

8. Kalman, R.E. Contribution to the theory of optimal control. Boletin de la Sociedad Matematica Mexicana 1960, 5, 102-119.

9. Bylov, B.F.; Vinograd, R.E.; Grobman, D.M.; Nemytskii, V.V. Theory of Lyapunov Exponents; Nauka: Moscow, Russia, 1966.

10. Demidovich, B.P. Lectures on the Mathematical Stability Theory; Nauka: Moscow, Russia, 1967.

11. Barreira, L. Lyapunov Exponents; Birkhäuser: Cham, Switzerland, 2017. [CrossRef]

12. Makarov, E.K.; Popova, S.N. Controllability of Asymptotic Invariants of Time-Dependent Linear Systems; Belaruskaya Navuka: Minsk, Belarus, 2012.

13. Makarov, E.K.; Popova, S.N. Global controllability of central exponents of linear systems. Russ. Math. 1999, 43, 56-63.

14. Makarov, E.K.; Popova, S.N. The global controllability of a complete set of Lyapunov invariants for two-dimensional linear systems. Differ. Equ. 1999, 35, 97-107.

15. Popova, S.N. Global controllability of the complete set of Lyapunov invariants of periodic systems. Differ. Equ. 2003, 39, 1713-1723. [CrossRef]

16. Popova, S.N. Global reducibility of linear control systems to systems of scalar type. Differ. Equ. 2004, 40, 43-49. [CrossRef]

17. Popova, S.N. On the global controllability of Lyapunov exponents of linear systems. Differ. Equ. 2007, 43, 1072-1078. [CrossRef]

18. Babiarz, A.; Banshchikova, I.; Czornik, A.; Makarov, E.; Niezabitowski, M.; Popova, S. On assignability of Lyapunov spectrum of discrete linear time-varying system with control. In Proceedings of the 2016 21st International Conference on Methods and Models in Automation and Robotics (MMAR), Miedzyzdroje, Poland, 29 August-1 September 2016. [CrossRef]

19. Babiarz, A.; Czornik, A.; Makarov, E.; Niezabitowski, M.; Popova, S. Pole placement theorem for discrete time-varying linear systems. SIAM J. Control Optim. 2017, 55, 671-692. [CrossRef] 
20. Popova S. Assignability of certain Lyapunov invariants for linear discrete-time systems. IFAC-PapersOnLine 2018, 51, 40-45. [CrossRef]

21. Babiarz, A.; Banshchikova, I.; Czornik, A.; Makarov, E.; Niezabitowski, M.; Popova, S. Necessary and sufficient conditions for assignability of the Lyapunov spectrum of discrete linear time-varying systems. IEEE Trans. Autom. Control 2018, 63, 3825-3837. [CrossRef]

22. Babiarz, A.; Banshchikova, I.; Czornik, A.; Makarov, E.; Niezabitowski, M.; Popova, S. Proportional local assignability of Lyapunov spectrum of linear discrete time-varying systems. SIAM J. Control Optim. 2019, 57, 1355-1377. [CrossRef]

23. Babiarz, A.; Banshchikova, I.; Czornik, A.; Makarov, E.; Niezabitowski, M.; Popova, S. Assignability of Lyapunov spectrum for discrete linear time-varying systems. Springer Proc. Math. Stat. 2020, 312, 133-147. [CrossRef]

24. Barabanov, E.A.; Konyukh, A.V. Bohl exponents of linear differential systems. Mem. Differ. Equ. Math. Phys. 2001, 24, 151-158.

25. Bykov, V.V. Bohl exponents and Baire classes of functions. J. Math. Sci. 2015, 210, 168-185. [CrossRef]

26. Bykov, V.V. Baire classes of Lyapunov invariants. Sb. Math. 2017, 208, 620-643. [CrossRef]

27. Barabanov, E.; Vaidzelevich, A.; Czornik, A.; Niezabitowski, M. Exact boundaries of mobility of the Lyapunov, Perron and Bohl exponents of linear difference systems under small perturbations of the coefficient matrices. AIP Conf. Proc. 2016, 1738, 130007. [CrossRef]

28. Czornik, A.; Konyukh, A.; Niezabitowski, M. Which functions may be the upper Bohl function of the diagonal discrete linear time-varying systems? J. Math. Anal. Appl. 2017, 452, 1420-1433. [CrossRef]

29. Babiarz, A.; Czornik, A.; Konyukh, A.; Niezabitowski, M. What types of functions may be the lower Bohl function of a diagonal discrete linear time-varying systems? J. Frankl. Inst. 2017, 354, 5131-5144. [CrossRef]

30. Babiarz, A.; Czornik, A.; Niezabitowski, M. On the assignability of regularity coefficients and central exponents of discrete linear time-varying systems. IFAC-PapersOnLine 2019, 52, 64-69. [CrossRef]

31. Babiarz, A.; Czornik, A.; Niezabitowski, M. Relations between Bohl exponents and general exponent of discrete linear time-varying systems. J. Differ. Equ. Appl. 2019, 25, 560-572. [CrossRef]

32. Czornik, A.; Konyukh, A.; Konyukh, I.; Niezabitowski, M.; Orwat, J. On Lyapunov and upper Bohl exponents of diagonal discrete linear time-varying systems. IEEE Trans. Autom. Control 2019, 64, 5171-5174. [CrossRef]

33. Pinto, N.; Robledo, G. Some relations between Bohl exponents and the exponential dichotomy spectrum. J. Differ. Equ. Appl. 2019, 25, 573-582. [CrossRef]

34. Datko, R. A linear control problem in an abstract Hilbert space. J. Differ. Equ. Appl. 1971, 9, $346-359$. [CrossRef]

35. Slemrod, M. The linear stabilization problem in Hilbert space. J. Funct. Anal. 1972, 11, 334-345. [CrossRef]

36. Russell, D.L. Controllability and stabilizability theory for linear partial differential equations: Recent progress and open questions. SIAM Rev. 1978, 20, 639-739. [CrossRef]

37. Pritchard, A.J.; Zabczyk, J. Stability and stabilizability of infinite-dimensional systems. SIAM Rev. 1981, 23, 25-52. [CrossRef]

38. Maciej Przyłuski, K.; Rolewicz, S. On stability of linear time-varying infinite-dimensional discrete-time systems. Syst. Control Lett. 1984, 4, 307-315. [CrossRef]

39. Logemann, H. Stability and stabilizability of linear infinite-dimensional discrete-time systems. IMA J. Math. Control Inf. 1992, 9, 255-263. [CrossRef]

40. Luo, Z.-H.; Guo, B.-Z.; Morgu, O. Stability and Stabilization of Infinite Dimensional Systems with Applications; Springer: London, UK, 1999. [CrossRef]

41. Raymond, J.-P. Stabilizability of infinite dimensional systems by finite dimensional control. Comput. Methods Appl. Math. 2019, 19, 267-282. [CrossRef]

42. Barreira, L.; Valls, C. Stability of the Lyapunov exponents under perturbations. Ann. Funct. Anal. 2017, 8, 398-410. [CrossRef]

43. Barreira, L.; Dragičević, D.; Valls, C. Nonuniform spectrum on Banach spaces. Adv. Math. 2017, 321, 547-591. [CrossRef]

44. Barreira, L.; Dragičević, D.; Valls, C. Spectrum for compact operators on Banach spaces. J. Math. Soc. Jpn. 2019, 71, 1-17. [CrossRef]

45. Daleckii, J.L.; Krein, M.G. Stability of Solutions of Differential Equations in Banach Space; American Mathematical Society: Providence, RI, USA, 1974. 
46. Makarov, E.K. On the discreteness of asymptotic invariants of linear differential systems. Differ. Equ. 1998, 34, 1323-1331.

47. Triggiani, R. Controllability and observability in Banach space with bounded operators. SIAM J. Control 1975, 13, 462-491. [CrossRef]

48. Zaitsev, V.A. Lyapunov reducibility and stabilization of nonstationary systems with an observer. Differ. Equ. 2010, 46, 437-447. [CrossRef]

49. Curtain, R.F.; Zwart, H. An Introduction to Infinite-Dimensional Linear Systems Theory; Springer: New York, NY, USA, 1995. [CrossRef]

50. Zabczyk, J. Mathematical Control Theory; Birkhäuser: Boston, MA, USA, 2008. [CrossRef]

51. Kolmogorov, A.N.; Fomin, S.V. Elements of the Theory of Functions and Functional Analysis; Graylock Press: Rochester, NY, USA, 1957.

(C) 2020 by the authors. Licensee MDPI, Basel, Switzerland. This article is an open access article distributed under the terms and conditions of the Creative Commons Attribution (CC BY) license (http://creativecommons.org/licenses/by/4.0/). 